\title{
Relativity in Clifford's Geometric Algebras of Space and Spacetime
}

\author{
William E. Baylis \\ Dept. of Physics, University of Windsor, Windsor, ON, Canada N9B 3P4 \\ Garret Sobczyk \\ Dept. de Física y Matemáticas, Universidad de las Américas, Cholula, Puebla, México 72820
}

\begin{abstract}
Of the various formalisms developed to treat relativistic phenomena, those based on Clifford's geometric algebra are especially well adapted for clear geometric interpretations and computational efficiency. Here we study relationships between formulations of special relativity in the spacetime algebra (STA) $C \ell_{1,3}$ of the underlying Minkowski vector space, and in the algebra of physical space (APS) $C \ell_{3}$. STA lends itself to an absolute formulation of relativity, in which paths, fields, and other physical properties have observer-independent representations. Descriptions in APS are related by a one-to-one mapping of elements from APS to the even subalgebra STA ${ }^{+}$of STA. With this mapping, reversion in APS corresponds to hermitian conjugation in STA. The elements of STA are all that is needed to calculate physically measurable quantities (called measurables) because only they entail the observer dependence inherent in any physical measurement. As a consequence, every relativistic physical process that can be modeled in STA also has a representation in APS, and vice versa. In the presence of two or more inertial observers, two versions of APS present themselves. In the absolute version, both the mapping to $\mathrm{STA}^{+}$and hermitian conjugation are observer dependent, and the proper basis vectors of any observer are persistent vectors that sweep out timelike planes in spacetime. To compare measurements by different inertial observers in APS, we express them in the proper algebraic basis of a single observer. This leads to the relative version of APS, which can be related to STA by assigning every inertial observer in STA to a single absolute frame in STA. The equivalence of inertial observers makes this permissible. The mapping and hermitian conjugation are then the same for all observers. Relative APS gives a covariant representation of relativistic physics with spacetime multivectors represented by multiparavectors in APS. We relate the two versions of APS as consistent models within the same algebra.
\end{abstract}




\section{INTRODUCTION}

Clifford's geometric algebra offers a powerful unifying language for the study of physics

1, 2, 3, 4, 5, 6, 7, 8, 9, 10]. Relativistic problems can be treated efficiently both in the spacetime algebra (STA) [4, 11], the Clifford algebra $C \ell_{1,3}$ of Minkowski spacetime, and in the algebra of physical space (APS) [3, 12, 13, 14], the Clifford algebra $\mathrm{Cl}_{3}$ of threedimensional Euclidean physical space. As real algebras, STA operates in a linear space of sixteen dimensions, whereas the linear space of APS has eight dimensions. APS can also be viewed as the 4-dimensional algebra of complex paravectors [15]. It is also isomorphic to complex quaternions, which have along history of applications in relativity, [16, 17] but whose geometrical interpretation is less obvious.

The connection between STA and APS highlights an important relation, only rarely explicitly expressed, between the basis vectors in Newtonian mechanics and those of special relativity. Newtonian basis vectors are usually viewed as unit displacement vectors in physical space that persist in time. Relativistically, such persistent vectors are associated with "timelike" bivectors (bivectors with positive squares, see below) representing planes that are swept out in spacetime by a given spatial direction with the passage of time. Under boosts, timelike bivectors transform into spacetime bivectors that have picked up spatial bivector parts (with negative squares), and the corresponding Newtonian basis vectors transform into a mixture of a spatial vector and a spatial bivector (a plane).

The formulation of relativity in STA can be characterized as absolute, in that physical paths, fields, and other properties of objects are expressed independent of any observer. The formulation in APS can be either absolute [15] or relative [12, 13, 14]. In both APS versions, the relationship between APS and $\mathrm{STA}^{+}$is expressed as an algebra isomorphism together with an operation called hermitian conjugation. Hermitian conjugation, identified with reversion in APS, separates elements of APS into real and imaginary parts, the real part consisting of scalars and vectors (elements of grade 0 and 1), and the imaginary part consisting of pseudoscalars (trivectors) and bivectors (elements of grade 3 and 2).

If we consider only one inertial observer, the two APS versions are equivalent. In the absolute APS approach, one posits a distinct absolute frame and hermitian conjugation for each observer, so that the reality and the grading of the elements of APS are observer dependent. The relative APS approach can be related to STA by assuming that all observers 
use a single absolute inertial frame in STA to form their proper basis. Since all inertial frames in STA are equivalent, each inertial observer can identify her frame with the chosen inertial frame. In this way, the same hermitian conjugation is used for all observers. The relative APS formulation admits a covariant formulation, of critical importance to physicists, in which real paravectors are naturally associated with spacetime vectors and higher paravector grades represent other covariant geometrical objects in spacetime. While its formulation and justification [5, 12] does not depend on STA, its connection to $\mathrm{STA}^{+}$clearly shows the relationship between the conceptually different relative and absolute approaches.

In the following sections, we first review APS and STA, and then construct APS from the even subalgebra $\mathrm{STA}^{+}$of STA. The formulation of APS is obtained initially for a single observer. This part is largely a review of previous work [11] but is included here for completeness. The connections become more convoluted when additional observers are added, as we investigate first in the absolute version of APS. We then see how, through the relation of measurable coefficients of covariant spacetime elements, we are led to the relative version. We can recover STA from APS by formulating relations in the proper basis of a single inertial observer in APS assigned to the absolute frame of STA. From these relationships we conclude that every physical process formulated in STA can equally well be described in APS.

\section{REVIEW OF APS}

We present here a brief summary of the use of APS to model relativistic phenomena. More details can be found elsewhere [5, 12]. The elements of APS are the real vectors $\mathbf{u}, \mathbf{v}, \mathbf{w}$ of physical space $\mathbb{R}^{3}$ and all their sums and products $\mathbf{u u}, \mathbf{u v}+\mathbf{u v w}, \ldots$ The elements of APS form an associative algebra under addition and multiplication. We will see later in the formulation of APS as $\mathrm{STA}^{+}$that the concept of a vector is itself, implicitly, relative to an observer.

The square of any vector $\mathbf{u} \in A P S$ is defined as its length squared :

$$
\mathbf{u}^{2} \equiv \mathbf{u u}=\mathbf{u} \cdot \mathbf{u}
$$

where $\mathbf{u} \cdot \mathbf{u}$ is the usual inner product. This axiom, together with the usual rules for adding and multiplying square matrices, determines the entire algebra. If we put $\mathbf{u}=\mathbf{v}+\mathbf{w}$, the 
axiom implies

$$
\mathbf{v} \mathbf{w}+\mathbf{w} \mathbf{v}=2 \mathbf{v} \cdot \mathbf{w}
$$

Evidently the algebra is not commutative and, in particular, the product of perpendicular vectors is anticommutative $\mathbf{u v}=-\mathbf{v u}$. It is called a bivector and has a geometric interpretation as the oriented plane containing $\mathbf{u}$ and $\mathbf{v}$.

Let $\left\{\mathbf{e}_{1}, \mathbf{e}_{2}, \mathbf{e}_{3}\right\}$ be an orthonormal basis of $\mathbb{R}^{3}$. The corollary (2) implies that

$$
\mathbf{e}_{j} \mathbf{e}_{k}+\mathbf{e}_{k} \mathbf{e}_{j}=2 \delta_{j k}
$$

where the Kronecker delta $\delta_{j k}$ gives the Euclidean metric of physical space. For example, $\mathbf{e}_{1}^{2}=1$ and $\mathbf{e}_{1} \mathbf{e}_{2}=-\mathbf{e}_{2} \mathbf{e}_{1}$. The bivector $\mathbf{e}_{1} \mathbf{e}_{2}$, a multivector of grade 2 , represents a directed area in the plane of the vectors. Its "direction" corresponds to circulation in the plane: if the circulation is reversed, the sign of the bivector is reversed. The bivector replaces the (Gibbs-Heaviside) vector cross product of polar vectors, but unlike the usual cross product, it is intrinsic to the plane and can be applied to planes in spaces of any number of dimensions.

The unit bivector $\mathbf{e}_{1} \mathbf{e}_{2}$ squares to

$$
\left(\mathbf{e}_{1} \mathbf{e}_{2}\right)^{2}=-\mathbf{e}_{2} \mathbf{e}_{1} \mathbf{e}_{1} \mathbf{e}_{2}=-1
$$

and generates rotations and reflections in the plane of $\mathbf{e}_{1}$ and $\mathbf{e}_{2}$. A general vector $\mathbf{v}$, with components both in the plane and perpendicular to it, is rotated through the angle $\phi$ in the $\mathbf{e}_{1} \mathbf{e}_{2}$ plane by

$$
\mathbf{v} \rightarrow R \mathbf{v} R^{\dagger}
$$

where the rotors $R, R^{\dagger}$ are

$$
\begin{aligned}
R & =\exp \left(-\mathbf{e}_{1} \mathbf{e}_{2} \phi / 2\right)=\cos \frac{\phi}{2}-\mathbf{e}_{1} \mathbf{e}_{2} \sin \frac{\phi}{2} \\
R^{\dagger} & =\cos \frac{\phi}{2}-\left(\mathbf{e}_{1} \mathbf{e}_{2}\right)^{\dagger} \sin \frac{\phi}{2}=\cos \frac{\phi}{2}-\mathbf{e}_{2} \mathbf{e}_{1} \sin \frac{\phi}{2}=R^{-1} .
\end{aligned}
$$

The dagger $\dagger$ used above denotes the conjugation of reversion, which reverses the order of vectors in all products. Thus, for any vector $\mathbf{v}, \mathbf{v}^{\dagger}=\mathbf{v}$, and the reversion of a product, say $A B$, of arbitrary elements can be found from $(A B)^{\dagger}=B^{\dagger} A^{\dagger}$. (A tilde ${ }^{\sim}$ is used in STA to indicate reversion, but when we associate APS with the even subalgebra $\mathrm{STA}^{+}$of STA we require distinct symbols.) In spaces of definite metric such as Euclidean spaces, one commonly represents the basis vectors by hermitian matrices. The dagger then corresponds 
to hermitian conjugation and can be used to split elements into "real" (hermitian) and "imaginary" (antihermitian) parts:

$$
\begin{aligned}
A & =\langle A\rangle_{\Re}+\langle A\rangle_{\Im} \\
\langle A\rangle_{\Re} & =\frac{A+A^{\dagger}}{2},\langle A\rangle_{\Im}=\frac{A-A^{\dagger}}{2} .
\end{aligned}
$$

The standard basis of APS over the reals can be specified by

$$
\left\{1, \mathbf{e}_{1}, \mathbf{e}_{2}, \mathbf{e}_{3}, \mathbf{e}_{2} \mathbf{e}_{3}, \mathbf{e}_{3} \mathbf{e}_{1}, \mathbf{e}_{1} \mathbf{e}_{2}, \mathbf{e}_{1} \mathbf{e}_{2} \mathbf{e}_{3}\right\}
$$

where the trivector $\mathbf{e}_{1} \mathbf{e}_{2} \mathbf{e}_{3}$ squares to -1. In APS, $\mathbf{e}_{1} \mathbf{e}_{2} \mathbf{e}_{3}$ is the volume element, also known as the unit pseudoscalar. It commutes with every vector and hence with all elements and can therefore be identified with the unit imaginary:

$$
\mathbf{e}_{1} \mathbf{e}_{2} \mathbf{e}_{3}=i
$$

The center of APS (the part that commutes with all elements) is spanned by $\{1, i\}$ and is identified with the complex field. Bivectors can be identified as imaginary vectors (pseudovectors) in APS. For example, $\mathbf{e}_{1} \mathbf{e}_{2}=\mathbf{e}_{1} \mathbf{e}_{2} \mathbf{e}_{3} \mathbf{e}_{3}=i \mathbf{e}_{3}$. We can now take the set

$$
\left\{1, \mathbf{e}_{1}, \mathbf{e}_{2}, \mathbf{e}_{3}\right\}
$$

as the standard basis of APS over the complex scalars.

The sum of a real scalar and a real vector is called a paravector. A typical paravector $p$ can be expanded

$$
p=p^{0}+\mathbf{p}=p^{\mu} \mathbf{e}_{\mu}
$$

where, for notational convenience in using the compact Einstein summation convention, we put $\mathbf{e}_{0}=1$. The convention is that repeated lower-case Greek indices are summed over $0,1,2,3$, whereas repeated lower-case Latin indices are summed over the spatial values 1, 2, 3 . Every element in APS can be expressed as a complex paravector. Reversion (the dagger conjugation) complex-conjugates the complex coefficients and thus changes the sign of the pseudoscalar and pseudovector parts. Real paravector space is a four-dimensional linear space spanned by the basis $\left\{\mathbf{e}_{0}, \mathbf{e}_{1}, \mathbf{e}_{2}, \mathbf{e}_{3}\right\}$ over the reals.

Just as for complex numbers, a natural quadratic form in paravector space is given by

$$
Q(p)=p \bar{p},
$$


where $\bar{p}=p^{0}-\mathbf{p}$ is called the Clifford conjugate of $p$. Clifford conjugation is extended to general elements $A, B$, of APS as an antiautomorphism: $\overline{A B}=\bar{B} \bar{A}$. It conveniently splits elements into scalarlike (S) and vectorlike (V) parts:

$$
\begin{aligned}
A & =\langle A\rangle_{S}+\langle A\rangle_{V} \\
\langle A\rangle_{S} & =\frac{A+\bar{A}}{2},\langle A\rangle_{V}=\frac{A-\bar{A}}{2} .
\end{aligned}
$$

The quadratic form (12) is scalarlike. Through it, paravector space inherits from the Euclidean metric of the underlying space of spatial vectors an inner product with the Minkowski spacetime metric:

$$
(p, q)=\langle p \bar{q}\rangle_{S}=\frac{p \bar{q}+q \bar{p}}{2}=p^{\mu} q^{\nu} \eta_{\mu \nu}
$$

with the metric tensor

$$
\eta_{\mu \nu}=\left\langle\mathbf{e}_{\mu} \overline{\mathbf{e}}_{\nu}\right\rangle_{S}=\left\{\begin{array}{c}
1, \mu=\nu=0 \\
-1, \mu=\nu=1,2,3 . \\
0, \mu \neq \nu
\end{array}\right.
$$

The appearance of the Minkowski spacetime metric suggests the use of real paravectors to model vectors in four-dimensional spacetime. Oriented planes in spacetime are then modeled by biparavectors [12] such as $\langle p \bar{q}\rangle_{V}=p^{\mu} q^{\nu}\left\langle\mathbf{e}_{\mu} \overline{\mathbf{e}}_{\nu}\right\rangle_{V}$, which represents the plane containing all linear combinations of the real paravectors $p$ and $q$, and which generally has both real (vector) and imaginary (bivector) parts. Rotations in paravector space are generated by biparavectors and leave the quadratic form (and hence inner products) of paravectors invariant. They represent physical Lorentz transformations. Rotations of the paravector $p$ in a single spacetime plane have the form

$$
p \rightarrow L p L^{\dagger}
$$

where $L$ is a Lorentz rotor of the form $L=\exp (\mathbf{W} / 2)$ and $\mathbf{W}$ is a biparavector for the plane of rotation. In the special case that $\mathbf{W}$ is imaginary, $L$ is a spatial rotation, and in the special case that it is real, $L$ is a boost (or hyperbolic rotation). More generally, any Lorentz rotor $L$ can be factored into the product $L=B R$ of a spatial rotation $R$ and a boost $B$. This formulation is pursued below in the relative formulation of APS. 


\section{REVIEW OF STA}

STA, introduced by Hestenes [1, 11], is the geometric algebra $C \ell_{1,3}$ of Minkowski spacetime. Minkowski spacetime has a pseudo-Euclidean metric that highlights the intrinsic difference between time and space. The aspects of STA presented here are those needed in our discussion below. Since APS can be equated to the even subalgebra of STA, particular attention is paid to this subalgebra. Each abstract inertial frame of STA consists of a constant 4-dimensional orthonormal vector basis $\left\{\gamma_{0}, \gamma_{1}, \gamma_{2}, \gamma_{3}\right\} \equiv\left\{\gamma_{\mu}\right\}$ satisfying

$$
\gamma_{\mu} \gamma_{\nu}+\gamma_{\nu} \gamma_{\mu}=2 \eta_{\mu \nu}
$$

where $\eta_{\mu \nu}$ are elements (16) of the Minkowski spacetime metric tensor.

The history (worldline) of an idealized point particle $P$ is a timelike curve $r^{P}\left(\tau^{P}\right)$ giving its position in STA as a function of a scalar parameter $\tau^{P}$, which we take to be its proper time. The tangent vector $u^{P}=d r^{P} / d \tau^{P}$ is its proper velocity (in units with $c=1$ ). In a commoving frame (an inertial frame instantaneously moving with the observer) $\left\{\gamma_{\mu}^{P}\right\}$ of the particle, the displacement has only a time component:

$$
d r^{P}=\gamma_{0}^{P} d \tau^{P}
$$

and the commoving unit time axis $\gamma_{0}^{P}$ is thus seen to be coincident with the proper velocity: $u^{P}=\gamma_{0}^{P}$.

The spacetime curve $r^{P}\left(\tau^{P}\right)$ and its tangent vector $u^{P}$ are abstract and do not, by themselves, determine physically measurable values. They are independent of the observer and therefore unchanged by passive transformations (transformations of the observer). For this reason Hestenes [4] calls such spacetime quantities invariant. However, they do transform under active Lorentz rotations, and to avoid possible confusion with Lorentz scalars, which are invariant under both passive and active transformations, we prefer to call them absolute. Physically measurable quantities ("measurables"), on the other hand, are either Lorentz invariants or are derived from vector components relative to the observer. As we show below, these are determined by even elements of STA.

An inertial observer, say Alice, can be idealized as a congruence of parallel histories $r^{A}\left(\tau^{A}\right)$, all with the same given proper velocity $u^{A}=d r^{A} / d \tau^{A}$ with $d u^{A} / d \tau^{A}=0$ and a

constant commoving frame $\left\{\gamma_{\mu}^{A}\right\}$ with $\gamma_{0}^{A}=u^{A}$. When Alice measures physical quantities 
represented by geometric objects such as vectors, she normally expresses them in her frame $\left\{\gamma_{\mu}^{A}\right\}$ of spacetime basis vectors. The $\gamma_{\mu}^{A}$ are abstract, and it is the scalar coefficients of the expansion that constitute the measurables for Alice. A different observer, say Bob, has his own frame $\left\{\gamma_{\mu}^{B}\right\}$ with $\gamma_{0}^{B}=u^{B}$ that he normally uses for measurements. We assume that the handedness of the two frames is the same:

$$
\gamma_{0}^{A} \gamma_{1}^{A} \gamma_{2}^{A} \gamma_{3}^{A}=\gamma_{0}^{B} \gamma_{1}^{B} \gamma_{2}^{B} \gamma_{3}^{B} \equiv \mathbf{I}
$$

where the pseudoscalar I of STA anticommutes with vectors.

Any two inertial frames of the same handedness are related by a Lorentz rotation, also known as a restricted (proper orthochronous) Lorentz transformation, and is specified by a rotor $L$. Every Lorentz rotation can be expressed as the product of a spatial rotation and a boost (a velocity transformation). There is in particular a rotor $L$ that relates Alice's frame to Bob's:

$$
\gamma_{\mu}^{B}=L \gamma_{\mu}^{A} \tilde{L}
$$

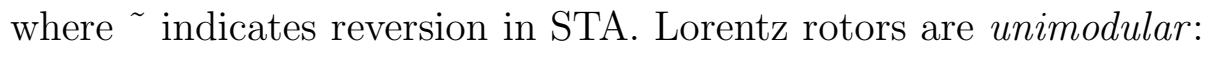

$$
L \tilde{L}=1
$$

and consequently all products of spacetime vectors transform in the same way in STA. For $\mu=0$, the transformation (19) relates the proper velocities

$$
\gamma_{0}^{B}=u^{B}=L \gamma_{0}^{A} \tilde{L}=L u^{A} \tilde{L} .
$$

It is common to define hermitian conjugation of any element $K$ for Alice by

$$
K^{\dagger A}=\gamma_{0}^{A} \tilde{K} \gamma_{0}^{A}
$$

The conjugation symbol $\dagger A$ is used here instead of the more usual $\dagger$ to underscore its dependence on the observer's frame. In terms of the hermitian conjugate, the proper velocities are related by

$$
\begin{aligned}
\gamma_{0}^{B} & =u^{B}=L L^{\dagger A} \gamma_{0}^{A}=L L^{\dagger A} u^{A} \\
L L^{\dagger A} & =\gamma_{0}^{B} \gamma_{0}^{A}=u^{B} u^{A},
\end{aligned}
$$

where we noted from (18) that $\left(\gamma_{0}^{A}\right)^{2}=1$. If the frames of Alice and Bob are related by a pure spatial rotation, their time axes and hence proper velocities $u^{B}$ and $u^{A}$ are equal 
and $L^{\dagger A}=\tilde{L}=L^{\dagger B}$. On the other hand, if their frames are related by a pure boost, then $L^{\dagger A}=L=L^{\dagger B}$ and $L^{2}=\gamma_{0}^{B} \gamma_{0}^{A}=u^{B} u^{A}$. In the most general case, the Lorentz rotor $L$ is the product of boost and spatial-rotation rotors, and $L L^{\dagger A}$ is simply the square of the boost rotor.

A boost along the $\gamma_{1}^{A}$ direction is a Lorentz rotation generated by the STA bivector $\gamma_{10}^{A} \equiv \gamma_{1}^{A} \gamma_{0}^{A}$, and it has the form $\exp \left(w \gamma_{10}^{A} / 2\right)$, where the scalar parameter $w$ is called the rapidity of the boost. We can expand the exponential to get for the rotor $L$

$$
L^{2}=\exp \left(w \gamma_{10}^{A}\right)=\gamma(1+\mathbf{v})=u^{B} u^{A}
$$

where $\gamma \equiv \cosh w$ is the Lorentz time dilation factor between the observers, and $\mathbf{v}=$ $\gamma_{10}^{A} \tanh w$ gives the relative coordinate velocity of Bob with respect to Alice. The coordinate velocity of Alice as seen by Bob is $\mathbf{- v}$. The plane of the Lorentz rotation is itself invariant under $L: \gamma_{10}^{B}=L \gamma_{10}^{A} \tilde{L}=\gamma_{10}^{A}$, and $\gamma$ and $|\mathbf{v}|$ are measurables for both Alice and Bob.

We want to compare measurements made by Alice and Bob of an absolute spacetime vector, which for concreteness we take to be the spacetime position $r$. (Since $r$ depends on the origin of coordinates, in order that this transform under fixed $L$ as a spacetime vector, we must assume that the origins of Alice's and Bob's frames coincide. Alternatively, we could replace $r$ by an affine spacetime vector.) Thus, $r$ might be one point on the history of a point particle or some other event. It can be expanded in the basis vectors of any inertial frame, for example in the frame $\left\{\gamma_{\mu}^{A}\right\}$ of Alice:

$$
r=r_{A}^{\mu} \gamma_{\mu}^{A}
$$

As emphasized above, the spacetime vectors $r$ and $\gamma_{\nu}^{A}$ are abstract and not directly measurable; it is the scalar coefficients $r_{A}^{\mu}$ that Alice can measure. The time component of $r$ measured by Alice is

$$
t_{A} \equiv r_{A}^{0}=r \cdot \gamma_{0}^{A}
$$

More generally, the spacetime vector $r$ relative to Alice is given by the expansion

$$
r \gamma_{0}^{A}=r_{A}^{\mu} \gamma_{\mu}^{A} \gamma_{0}^{A} \equiv r_{A}=t_{A}+\mathbf{r}_{A}
$$

where the spacetime bivector $\mathbf{r}_{A}=r \wedge \gamma_{0}^{A}=r_{A}^{k} \gamma_{k 0}^{A} \in \mathrm{STA}^{+}$, with $\gamma_{k 0}^{A} \equiv \gamma_{k}^{A} \gamma_{0}^{A}$, is interpreted in APS as the spatial position vector from the origin to the particle that Alice measures. 
The even element $r_{A}=r \gamma_{0}^{A}$ is called the relative position, and its expansion (27) is called a space-time split [4, 11].

Analogous relations can be written for Bob's measurables. The relative positions $r_{A}=$ $r \gamma_{0}^{A}$ and $r_{B}=r \gamma_{0}^{B}$ are related by the passive Lorentz rotation

$$
\begin{aligned}
r_{B} & =r \gamma_{0}^{B}=r L \gamma_{0}^{A} \tilde{L}=r \gamma_{0}^{A} \tilde{L}^{\dagger A} \tilde{L} \\
& =r_{A} \tilde{L}^{\dagger A} \tilde{L}
\end{aligned}
$$

The inverse transformation can be written

$$
r_{A}=r_{B} L L^{\dagger A}=r_{B} L^{\dagger B} L
$$

where $L^{\dagger B}=\gamma_{0}^{B} \tilde{L} \gamma_{0}^{B}=L \gamma_{0}^{A} \tilde{L} \tilde{L} L \gamma_{0}^{A} \tilde{L}=L L^{\dagger A} \tilde{L}$.

A different form of transformation results by boosting the event $r$ for a given observer. This is an example of an active transformation, in which the observer is fixed and the event is transformed. Let

$$
r^{\prime}=\operatorname{Lr} \tilde{L}
$$

This boost changes the relative position with respect to Alice according to

$$
\begin{aligned}
r_{A}^{\prime} & =r^{\prime} \gamma_{0}^{A}=r_{A}^{\prime \mu} \gamma_{\mu}^{A} \gamma_{0}^{A}=L r \tilde{L} \gamma_{0}^{A}=L r \gamma_{0}^{A} L^{\dagger A} \\
& =L r_{A} L^{\dagger A} .
\end{aligned}
$$

If both of these transformations are performed together (the order is not important when the same rotor is used for both) we obtain the relative spacetime position of the transformed event with respect to Bob:

$$
\begin{aligned}
r_{B}^{\prime} & =r^{\prime} \gamma_{0}^{B}=L r \gamma_{0}^{A} \tilde{L}=L r_{A}^{\mu} \gamma_{\mu}^{A} \gamma_{0}^{A} \tilde{L} \\
& =L r_{A} \tilde{L} .
\end{aligned}
$$

The three transformations of measurables (28), (31), (32) have distinct forms. In the transformation (32), in which both the observer and the event are boosted by the same amount, the unimodularity of $L$ (20) implies that the time measurements are the same: $t_{B}^{\prime}=t_{A}$, but the positions generally differ

$$
\mathbf{r}_{B}^{\prime}=r_{B}^{\prime k} \gamma_{k 0}^{B}=L \mathbf{r}_{A} \tilde{L}=L r_{A}^{j} \gamma_{j 0}^{A} \tilde{L}
$$

How are the three transformations to be interpreted physically? 


\section{A. Measurables in STA}

To understand the physical interpretation of the above equations, it is important to note that all of the measurables have been expressed in terms of elements of $\mathrm{STA}^{+}$, the even subalgebra of STA, spanned by the basis $\left\{1, \gamma_{\mu \nu}, I\right\}$, where the basis bivectors in STA are $\gamma_{\mu \nu} \equiv \frac{1}{2}\left(\gamma_{\mu} \gamma_{\nu}-\gamma_{\nu} \gamma_{\mu}\right)$ with $0 \leq \mu<\nu \leq 3$. The reason for this is that every physical measurement of a vector is relative and involves two absolute spacetime vectors: the absolute vector of the event or property being measured and a basis vector of the reference frame used by the observer. The results of the measurement depend on the orientation and motion of one with respect to the other.

Of course it is most common for an observer to employ a reference frame at relative rest. Measurements can then be expressed in $\mathrm{STA}^{+}$in the observer-dependent proper basis

$$
\left\{\boldsymbol{\sigma}_{\mu}^{A}=\gamma_{\mu}^{A} \gamma_{0}^{A}\right\},\left\{\boldsymbol{\sigma}_{\mu}^{B}=\gamma_{\mu}^{B} \gamma_{0}^{B}\right\}
$$

The bases are called proper because they are based on the absolute frames that are at rest with respect to the observer. The basis element representing the time axis in the relative frame is in each case unity:

$$
\boldsymbol{\sigma}_{0}^{A}=1=\boldsymbol{\sigma}_{0}^{B}
$$

and the spatial elements are spacetime bivectors $\boldsymbol{\sigma}_{k}=\gamma_{k 0}=\gamma_{k} \gamma_{0}$ representing planes containing both the spatial direction $\gamma_{k}$ and the time direction $\gamma_{0}$. These are the planes swept out by the unit spatial vector $\gamma_{k}$ in one unit of time. They are the spacetime planes representing persistent spatial vectors in physical space. The relation between the relative basis vectors is

$$
\boldsymbol{\sigma}_{\mu}^{B}=L \boldsymbol{\sigma}_{\mu}^{A} \tilde{L}
$$

for $\mu=0,1,2,3$, which follows directly from $\gamma_{\mu}^{B}=L \gamma_{\mu}^{A} \tilde{L}$ and $L \tilde{L}=1$. We will return to the meaning of this transformation below.

In terms of these proper bases, the three transformations (2813132) are

$$
\begin{aligned}
& r_{A} \rightarrow r_{B}=r_{B}^{\mu} \boldsymbol{\sigma}_{\mu}^{B}=r_{A} \tilde{L}^{\dagger A} \tilde{L} \text { passive } \\
& r_{A} \rightarrow r_{A}^{\prime}=r_{A}^{\prime \mu} \boldsymbol{\sigma}_{\mu}^{A}=L r_{A} L^{\dagger A} \text { active } \\
& r_{A} \rightarrow r_{B}^{\prime}=r_{B}^{\prime \mu} \boldsymbol{\sigma}_{\mu}^{B}=L r_{A} \tilde{L} \quad \text { both. }
\end{aligned}
$$

The measurables for Alice are the scalar coefficients $r_{A}^{\mu}$ and $r_{A}^{\prime \mu}$, and for Bob, they are $r_{B}^{\mu}$ and $r_{B}^{\prime \mu}$. We can relate them using the orthogonality of the basis vectors. For example, for 
the passive case,

$$
r_{B}^{\mu}=r_{A}^{\nu}\left\langle\boldsymbol{\sigma}_{\nu}^{A} \tilde{L}^{\dagger A} \tilde{L} \boldsymbol{\sigma}_{\mu}^{B}\right\rangle_{S}=r_{A}^{\nu}\left\langle\boldsymbol{\sigma}_{\nu}^{A} \tilde{L}^{\dagger A} \boldsymbol{\sigma}_{\mu}^{A} \tilde{L}\right\rangle_{S}
$$

However, the relations are most transparently seen if the transformations (36) are expressed in terms of a proper basis of a single observer. If we insert the transformation (35) into (36), we find

$$
\begin{aligned}
r_{B}^{\mu} \boldsymbol{\sigma}_{\mu}^{A}=r_{A}^{\nu} \tilde{L} \boldsymbol{\sigma}_{\nu}^{A} \tilde{L}^{\dagger A}=\tilde{L} r_{A} \tilde{L}^{\dagger A} & \text { passive } \\
r_{A}^{\prime \mu} \boldsymbol{\sigma}_{\mu}^{A}=r_{A}^{\nu} L \boldsymbol{\sigma}_{\nu}^{A} L^{\dagger A}=L r_{A} L^{\dagger A} & \text { active } \\
r_{B}^{\prime \mu} \boldsymbol{\sigma}_{\mu}^{A}=r_{A}^{\nu} \boldsymbol{\sigma}_{\nu}^{A}=r_{A} & \text { both. }
\end{aligned}
$$

Note that the active and passive transformations (38) have similar forms but with inverse rotors. They demonstrate in particular that under either active or passive boosts, time and space components are mixed and time intervals (and hence clock rates) change. The last relation of (38) shows that when both the event and the observer are transformed by the same rotor, the components are unchanged:

$$
r_{B}^{\prime \mu}=r_{A}^{\mu}
$$

In other words, the spacetime vector $r^{\prime}$ measured by Bob has the same components as $r$ measured by Alice.

Notice that $\boldsymbol{\sigma}_{k}^{A}$ and $\boldsymbol{\sigma}_{k}^{B}$, for $k=1,2,3$, are timelike bivectors when expressed in the

frames $\left\{\gamma_{\mu}^{A}\right\}$ and $\left\{\gamma_{\mu}^{B}\right\}$, respectively, of STA. In the next section, we will reinterpret the $\boldsymbol{\sigma}_{k}^{A}$ to be the persistent vectors of a single, absolute inertial frame, and study the resulting geometric algebra, which is isomorphic to $\mathrm{Cl}_{3}$.

\section{APS AS STA ${ }^{+}$}

Since APS is isomorphic to the even subalgebra of STA, we can derive the relativistic formalism for APS by equating the basis vectors $\mathbf{e}_{k}$ of APS to the appropriate elements of $\mathrm{STA}^{+}$. For Alice, we have

$$
\mathbf{e}_{k}=\boldsymbol{\sigma}_{k}^{A}=\gamma_{k 0}^{A}, k=1,2,3
$$

These three orthonormal vectors satisfy the axiom (3) and generate both APS and STA . The identification (40) associates the three spatial basis vectors of APS with timelike bivectors of STA. This reinforces the concept of persistent vectors in APS that sweep out timelike 
planes in STA. Since $\sigma_{0}^{A}=1$, we can extend the identification (40) to Alice's proper paravector basis $\left\{\mathbf{e}_{\mu}=\boldsymbol{\sigma}_{\mu}^{A}\right\}$ with

$$
\mathbf{e}_{\mu}=\boldsymbol{\sigma}_{\mu}^{A}=\gamma_{\mu}^{A} \gamma_{0}^{A}, \mu=0,1,2,3 .
$$

From this identification, the volume elements of APS and STA are

$$
i=\mathbf{e}_{1} \mathbf{e}_{2} \mathbf{e}_{3}=\boldsymbol{\sigma}_{1}^{A} \boldsymbol{\sigma}_{2}^{A} \boldsymbol{\sigma}_{3}^{A}=\gamma_{10}^{A} \gamma_{20}^{A} \gamma_{30}^{A}=\gamma_{0}^{A} \gamma_{1}^{A} \gamma_{2}^{A} \gamma_{3}^{A}=\mathbf{I} .
$$

As in STA, the volume element of APS squares to -1, but whereas in STA I anticommutes with all vectors, in both APS and $\mathrm{STA}^{+}$the volume element is part of the center of the algebra, that is, it commutes with all elements.

Clifford conjugation in APS corresponds to reversion in $\mathrm{STA}^{+}$

$$
\begin{aligned}
& \overline{\mathbf{e}}_{0}=\mathbf{e}_{0}=1=\tilde{1} \\
& \overline{\mathbf{e}}_{k}=-\mathbf{e}_{k}=-\gamma_{k 0}^{A}=\tilde{\gamma}_{k 0}^{A},
\end{aligned}
$$

and it follows that Clifford conjugation of an observer's proper basis is the same for every observer. Reversion in APS is equivalent to Alice's hermitian (dagger) conjugation (22) in $\mathrm{STA}^{+}$, and it follows that the proper basis vectors $\mathbf{e}_{\mu}$ are real as seen by Alice:

$$
\mathbf{e}_{\mu}^{\dagger}=\boldsymbol{\sigma}_{\mu}^{\dagger A}=\gamma_{0}^{A} \tilde{\boldsymbol{\sigma}}_{\mu}^{A} \gamma_{0}^{A}=\mathbf{e}_{\mu} .
$$

The equations (36) for Lorentz rotations in $\mathrm{STA}^{+}$are unchanged in APS except that, for Alice, $\boldsymbol{\sigma}_{\mu}^{A}$ is replaced by $\mathbf{e}_{\mu}$. The proper basis vectors $\boldsymbol{\sigma}_{\mu}^{B}$ used by Bob, given by (35), are seen by Alice to be complex. This can be understood, as mentioned above, as the result of transforming the timelike planes swept out by persistent vectors in time, and it reflects the action of the six-parameter group $S L(2, \mathbb{C})$ of Lorentz rotors, which mix timelike and spacelike planes in spacetime. Such planes correspond respectively to vectors and bivectors in APS. The fact that Alice sees Bob's frame as complex presents no physical problem because the definition of proper hermitian conjugation (22) depends on the observer and ensures that each observer sees her own proper basis vectors as real [18].

Indeed the mapping (41) between APS and $\mathrm{STA}^{+}$is observer dependent, and while Alice takes the proper basis vectors $\mathbf{e}_{k}$ of APS to be her timelike bivectors $\boldsymbol{\sigma}_{k}^{A}$ in $\mathrm{STA}^{+}$, Bob equates them to his bivectors $\boldsymbol{\sigma}_{k}^{B}$. Just as under Alice's hermitian conjugation, Bob's proper basis vectors $\boldsymbol{\sigma}_{k}^{B}$ are complex whereas hers are real, under Bob's conjugation, it is Alice's $\boldsymbol{\sigma}_{k}^{A}$ 
that are complex and his are real. By allowing both the mapping between APS and STA and hermitian conjugation to vary with observer, this formulation of APS is thus able to treat all observers on an equal footing. The proper vector basis used by any observer in this formulation, like the bivector basis in STA to which it is equated, is absolute and distinct from the bases used by other observers who are in relative motion. We therefore refer to this formulation of APS as absolute.

The important relations in relativity, however, are not the way one observer sees another observer's proper basis, but how the real, scalar values measured by one observer are related to those measured by another. Such relations are given by the transformations (38), and these suggest a relative formulation of APS.

\section{A. Covariant Multiparavectors}

While any collection of real vector components on the basis $\left\{\mathbf{e}_{0}, \mathbf{e}_{1}, \mathbf{e}_{2}, \mathbf{e}_{3}\right\}$ defines a real paravector, only some such collections transform together under Lorentz rotations as a spacetime vector. Those that do are said to be covariant. Furthermore, a spatial vector in APS may be part of a spacetime vector, whose components transform according to (38), or it may be part of a spacetime bivector, whose components transform differently. For example, the proper acceleration $d u / d \tau$ of a point particle is a spatial vector in the commoving inertial frame, and it transforms as in (38) above. However, the electric field $\mathbf{E}$ is also a pure vector, but it transforms distinctly. In fact, it transforms as a persistent vector and may be seen by a different observer as having both real vector (electric) and imaginary vector or bivector (magnetic) parts. Its basis expansion in APS is simply

$$
\mathbf{E}=E_{A}^{k} \boldsymbol{\sigma}_{k}^{A}
$$

for Alice, and naively applying the arguments made for the position vector $r_{A}$, it would be seen by Bob (using his proper conjugation) to be real and to pick up a scalar part. It would remain real because Bob's proper conjugation is different from Alice's, and the difference exactly compensates the generation of the imaginary part.

This, however, is wrong. There is no meaning to a scalar part of the electromagnetic field, and Bob really sees a magnetic (imaginary) component to the field. What is missing is a recognition that the proper acceleration and the electric-field vector belong to different types 
of covariant objects and therefore transform differently under Lorentz rotations. The proper acceleration is part of a spacetime vector whereas the electric field is part of a spacetime bivector $\mathbf{F}$ representing the electromagnetic field.

Alice and Bob see different electric and magnetic components of the given electromagnetic field $\mathbf{F}$ because their hermitian conjugations are different. Thus,

$$
\begin{gathered}
\mathbf{F}=\frac{1}{2}\left(\mathbf{F}+\mathbf{F}^{\dagger A}\right)+\frac{1}{2}\left(\mathbf{F}-\mathbf{F}^{\dagger A}\right)=\mathbf{E}_{A}+i \mathbf{B}_{A} \\
=\frac{1}{2}\left(\mathbf{F}+\mathbf{F}^{\dagger B}\right)+\frac{1}{2}\left(\mathbf{F}-\mathbf{F}^{\dagger B}\right)=\mathbf{E}_{B}+i \mathbf{B}_{B}
\end{gathered}
$$

To relate the measurables, that is the actual field components measured by Alice and Bob, we expand the covariant spacetime bivector of which it is a part in an inertial frame in STA and then express the result in a single proper basis of APS. For Alice the appropriate expansion is

$$
\begin{aligned}
\mathbf{F} & =\frac{1}{2} F_{A}^{\mu \nu} \gamma_{\mu \nu}^{A}=\frac{1}{4} F_{A}^{\mu \nu}\left(\gamma_{\mu 0}^{A} \gamma_{0 \nu}^{A}-\gamma_{\nu 0}^{A} \gamma_{0 \mu}^{A}\right) \\
& =\frac{1}{4} F_{A}^{\mu \nu}\left(\boldsymbol{\sigma}_{\mu}^{A} \tilde{\boldsymbol{\sigma}}_{v}^{A}-\boldsymbol{\sigma}_{\nu}^{A} \tilde{\boldsymbol{\sigma}}_{\mu}^{A}\right) .
\end{aligned}
$$

The same $\mathbf{F}$ can be expanded in Bob's basis $\mathbf{F}=\frac{1}{4} F_{B}^{\mu \nu}\left(\boldsymbol{\sigma}_{\mu}^{B} \tilde{\boldsymbol{\sigma}}_{\nu}^{B}-\boldsymbol{\sigma}_{\nu}^{B} \tilde{\boldsymbol{\sigma}}_{\mu}^{B}\right)$ so that the relation between the components seen by Alice and Bob is

$$
F_{B}^{\mu \nu}\left(\boldsymbol{\sigma}_{\mu}^{B} \tilde{\boldsymbol{\sigma}}_{\nu}^{B}-\boldsymbol{\sigma}_{\nu}^{B} \tilde{\boldsymbol{\sigma}}_{\mu}^{B}\right)=F_{A}^{\mu \nu}\left(\boldsymbol{\sigma}_{\mu}^{A} \tilde{\boldsymbol{\sigma}}_{v}^{A}-\boldsymbol{\sigma}_{\nu}^{A} \tilde{\boldsymbol{\sigma}}_{\mu}^{A}\right),
$$

where $\boldsymbol{\sigma}_{\mu}^{B} \tilde{\boldsymbol{\sigma}}_{\nu}^{B}-\boldsymbol{\sigma}_{\nu}^{B} \tilde{\boldsymbol{\sigma}}_{\mu}^{B}=L\left(\boldsymbol{\sigma}_{\mu}^{A} \tilde{\boldsymbol{\sigma}}_{v}^{A}-\boldsymbol{\sigma}_{\nu}^{A} \tilde{\boldsymbol{\sigma}}_{\mu}^{A}\right) \tilde{L}$. This leads to the relation

$$
F_{B}^{\mu \nu}\left(\boldsymbol{\sigma}_{\mu}^{A} \tilde{\boldsymbol{\sigma}}_{v}^{A}-\boldsymbol{\sigma}_{\nu}^{A} \tilde{\boldsymbol{\sigma}}_{\mu}^{A}\right)=4 \tilde{L} \mathbf{F} L=\tilde{L} F_{A}^{\mu \nu}\left(\boldsymbol{\sigma}_{\mu}^{A} \tilde{\boldsymbol{\sigma}}_{v}^{A}-\boldsymbol{\sigma}_{\nu}^{A} \tilde{\boldsymbol{\sigma}}_{\mu}^{A}\right) L
$$

under a passive Lorentz rotation. With the identification (41), in APS this becomes

$$
F_{B}^{\mu \nu}\left\langle\mathbf{e}_{\mu} \overline{\mathbf{e}}_{\nu}\right\rangle_{V}=\tilde{L} F_{A}^{\mu \nu}\left\langle\mathbf{e}_{\mu} \overline{\mathbf{e}}_{\nu}\right\rangle_{V} L
$$

Thus, whereas every observer sees the proper acceleration or (for inertial observers sharing a common spacetime origin) the position as a real paravector in APS, the boosted electric field becomes a mixture of real and imaginary vectors, representing the electric and magnetic fields seen by a different observer. Vectors transform differently depending on what (if any) type of covariant object they belong to. The ability to identify the type of covariant object being transformed is essential in establishing the correct transformations between the 
measurables (scalar coefficients) for different observers, and the use of covariant objects such as spacetime vectors and bivectors is also important for simplifying relations and bringing out the geometry and relativistic symmetries of the problem. In STA, covariant objects are generally homogenous $k$-vectors. In APS, homogeneous $k$-vectors are generally not covariant. Instead, it is the paravectors and multiparavectors that provide the covariant formulation. Their utility is based on the relative formulation of APS, discussed in the next Section.

\section{RELATIVE APS}

The formulation of relativity in absolute APS depends on the identification of the proper paravector basis and hermitian conjugation for each observer. Thus, Alice uses the basis $\left\{\boldsymbol{\sigma}_{\mu}^{A}\right\}$, which is real under her conjugation operator $\dagger A$, whereas Bob uses a different proper basis $\left\{\boldsymbol{\sigma}_{\mu}^{B}\right\}$ with the corresponding operator $\dagger B$. However, as seen above, to relate Alice's and Bob's measurables such as the scalar coefficients $r_{A}^{\mu}$ and $r_{B}^{\mu}$, we use transformations (38) that entail only one proper basis for both observers. In (38) we used Alice's proper basis and conjugation, but we would obtain the same results by using Bob's proper basis or, in fact, the proper basis of any inertial observer together with that observer's proper conjugation.

To see this, replace $\boldsymbol{\sigma}_{\mu}^{A}$ by $\tilde{L}_{C A} \boldsymbol{\sigma}_{\mu}^{C} L_{C A}$ in order to express the result in Carol's proper basis $\left\{\boldsymbol{\sigma}_{\mu}^{C}\right\}$. Here, $L_{C A}$ is the Lorentz rotor for the transformation from Alice to Carol. The passive transformation in (38), for example, becomes

$$
r_{A} \rightarrow r_{B}^{\mu} \tilde{L}_{C A} \boldsymbol{\sigma}_{\mu}^{C} L_{C A}=r_{A}^{\nu} \tilde{L} \tilde{L}_{C A} \boldsymbol{\sigma}_{\nu}^{C} L_{C A} \tilde{L}^{\dagger A}
$$

which since $L_{C A} \tilde{L}_{C A}=1$ and $\gamma_{0}^{C}=L_{C A} \gamma_{0}^{A} \tilde{L}_{C A}$, is equivalent to

$$
\begin{aligned}
r_{B}^{\mu} \boldsymbol{\sigma}_{\mu}^{C} & =r_{A}^{\nu} L_{C A} \tilde{L} \tilde{L}_{C A} \boldsymbol{\sigma}_{\nu}^{C} L_{C A} \tilde{L}^{\dagger A} \tilde{L}_{C A} \\
& =r_{A}^{\nu} L_{C A} \tilde{L} \tilde{L}_{C A} \boldsymbol{\sigma}_{\nu}^{C} \tilde{L}_{A C} \gamma_{0}^{A} L \gamma_{0}^{A} \tilde{L}_{C A} \\
& =r_{A}^{\nu} L_{C A} \tilde{L} \tilde{L}_{C A} \boldsymbol{\sigma}_{\nu}^{C} \gamma_{0}^{C} L_{C A} L \tilde{L}_{C A} \gamma_{0}^{C} \\
& =r_{A}^{\nu} \tilde{L}^{\prime} \boldsymbol{\sigma}_{\nu}^{C} \tilde{L}^{\prime \dagger C},
\end{aligned}
$$

where $L^{\prime}=L_{C A} L \tilde{L}_{C A}$ is the transformation from Alice to Bob as seen by Carol [12]. In the special case that Carol shares Alice's inertial frame, $L_{C A}=1$, whereas if Carol and Bob share the same frame, $L_{C A}=L$. 
The key point is that the transformation (44) of measurables has exactly the same form as the passive transformation in (38). The same result can be readily verified for the other transformations (38) of measurables. The transformations are the same no matter which inertial observer is used for the mapping of APS to $\mathrm{STA}^{+}$and for the definition of hermitian conjugation in $\mathrm{STA}^{+}$. Although the relation of absolute APS to $\mathrm{STA}^{+}$requires separate mappings and a separate hermitian conjugation for each inertial observer, this may be considered an artifact of the absolute approach in STA. Within relative APS it makes no difference which inertial observer is chosen; only one proper conjugation and one proper (relative) basis $\left\{\mathbf{e}_{\mu}\right\}$ are needed, and these can be associated with any inertial observer. The result can also be described as choosing one absolute frame in STA as the observer frame. Since all inertial frames are equivalent, any inertial observer can be assigned to this frame. Relative APS thus incorporates the basic principle of relativity that all inertial observers are equivalent and that only the relative motion and orientation of frames is physically significant.

When we organize the Lorentz transformations as in (38) to compute the measurables, such as the scalar components of a paravector $r_{A}$, we find the transformations of a spacetime vector, even in the special case when $r_{A}=\mathbf{e}_{\mu}$. We need to understand what it means for $\mathbf{e}_{\mu}=\boldsymbol{\sigma}_{\mu}^{A}=\gamma_{\mu}^{A} \gamma_{0}^{A}$, a bivector in $\mathrm{STA}^{+}$, to transform as a spacetime vector rather than as a bivector as in (35). The paravector transformation of $\mathbf{e}_{\mu}$ can be expressed in STA as

$$
\mathbf{e}_{\mu} \rightarrow L \mathbf{e}_{\mu} L^{\dagger A}=L \gamma_{\mu}^{A} \gamma_{0}^{A} L^{\dagger A}=\left(L \gamma_{\mu}^{A} \tilde{L}\right) \gamma_{0}^{A}
$$

where we have employed the definition (22) of Alice's proper conjugation. The paravector basis elements $\mathbf{e}_{\mu}$ are defined to be even elements of STA, and each involves the product of vectors from two absolute STA frames. The paravector transformation (45) transforms only one of the frames. The result helps to clarify the roles of the two factors: the first factor $\gamma_{\mu}^{A}$ gives the STA frame in which the observed object property is expanded, and the second factor $\gamma_{0}^{A}$ gives the absolute proper velocity of the observer. The two factors generally transform differently, as in the paravector transformation (45). The product of the two frame vectors gives the first relative to the second. An observer will normally choose a proper basis in which the object and observer frames are the same, but this is not necessary; she may also choose an object frame in relative motion. In all cases, in the relative version of APS the paravector basis elements are relative to the observer. 
To obtain the transformation for spacetime bivectors from that for paravectors, (45), we form a product of two paravectors and transform it:

$$
\mathbf{e}_{\mu}=\mathbf{e}_{\mu} \overline{\mathbf{e}}_{0} \rightarrow L \mathbf{e}_{\mu} L^{\dagger} \overline{\left(L \mathbf{e}_{0} L^{\dagger}\right)}=L \mathbf{e}_{\mu} \overline{\mathbf{e}}_{0} \bar{L}
$$

This is equivalent to the spacetime bivector transformation (35) of $\sigma_{\mu}^{A}$. Of course $\mathbf{e}_{0}=1$, but it is often convenient to add factors of $\mathbf{e}_{0}$ or $\overline{\mathbf{e}}_{0}$ to display the correct covariant behavior.

To summarize, in relative APS, spacetime vectors are covariantly represented by real paravectors. For example, the energy-momentum paravector of a particle is $p=m u=E+\mathbf{p}$. Multiparavectors of higher grade can be formed to represent other covariant geometrical objects, namely spacetime planes, hypersurfaces, and volumes. Simple Lorentz rotations of paravectors have the form (17) where $L$ is the exponential of a biparavector representing the plane of rotation. Since as discussed above, the spacetime vectors represented by paravectors in APS are all relative to the observer, a single Lorentz rotation can equally well represent an active transformation of the observed spacetime vector, the inverse passive transformation of the observer, or some combination thereof. In the passive case, the spacetime vector $p$ can be treated as invariant with respect to one observer, say Alice, and the transformation derived by expressing Bob's frame relative to Alice. Thus

$$
p_{A}=p_{A}^{\mu} \mathbf{e}_{\mu}=p_{B}^{\nu} \mathbf{u}_{\nu}
$$

where $\mathbf{u}_{\mu}=L \mathbf{e}_{\mu} L^{\dagger}$ is Bob's frame as seen by Alice. This gives $p_{B}^{\nu}=p_{A}^{\mu}\left\langle\mathbf{e}_{\mu} L \overline{\mathbf{e}}^{\nu} L^{\dagger}\right\rangle_{S}$, which is exactly the same relation as found from the transformation (17).

Multiparavectors of grades 0 through 4 form linear subspaces of the algebra. Grade0 paravectors are the same real scalars as grade-0 vectors, but covariantly they model spacetime scalars, which are invariant under Lorentz transformations. Grade-1 paravectors model spacetime vectors and form the four-dimensional paravector space that is also the direct sum of scalar and vector spaces. The biparavectors model spacetime planes and form a six-dimensional subspace from the direct sum of vector and bivector spaces. The four-dimensional subspace of triparavectors, which model hypersurfaces in spacetime, is the direct sum of bivector and trivector spaces. Finally, the paravector volume element, which models the spacetime pseudoscalar, also serves as the vector volume element:

$$
\mathbf{e}_{0} \overline{\mathbf{e}}_{1} \mathbf{e}_{2} \overline{\mathbf{e}}_{3}=\mathbf{e}_{1} \mathbf{e}_{2} \mathbf{e}_{3}=i
$$


The split of any paravector, biparavector or triparavector into its multivector parts is a space/time split in APS.

\section{DISCUSSION}

The measurement of a physical vector or multivector depends both on the vectors of the physical system to be measured and on the commoving frame of the observer. STA represents both the object to be measured and the observer in terms of abstract absolute frames, but the measurables are components of the object vectors on the basis vectors of the observer frame. These measurables appear as even elements of STA, involving products of vectors of the observed system with the frame vectors of the observer. If we consider only a single observer, we can map the elements of $\mathrm{STA}^{+}$onto those of APS in such a way that the basis elements $\mathbf{e}_{\mu}$ of APS are just proper relative basis elements $\boldsymbol{\sigma}_{\mu}=\gamma_{\mu} \gamma_{0}$ of the observer. Persistent spatial vectors of APS then correspond to timelike bivectors of STA, and hermitian conjugation, which gives reversion in APS, is defined using the proper velocity of the observer. The result is that paravectors in APS are always given relative to the observer. Only such relative paravectors and their products can be measured, and this is precisely what is computed in APS. The additional flexibility provided by STA, namely to compute absolute objects and observer frames independently, although at times conceptually appealing, is never needed in physical measurements. This is the reason that APS can represent any physical process as well as STA.

When there are two or more observers, they generally determine distinct values of the measurables, and an important goal of any relativistic formalism is to relate such values. In the absolute version of APS, the proper paravector basis $\left\{\mathbf{e}_{\mu}\right\}$, as well as the proper hermitian conjugation, depends on the observer and is identified with an absolute frame in $\mathrm{STA}^{+}$. It is the observer's own proper conjugation operator that tells her which parts of spacetime bivectors are her vectors and which are her bivectors. Lorentz boosts between different

observers mix spatial vectors and bivectors, all of which lie in the six-dimensional space of spacetime bivectors of $\mathrm{STA}^{+}$. Such mixing explains why boosts of persistent real vectors, such as the electric field, induce an observed magnetic field. A similar transformation applies to each observer's proper basis vectors when these are treated as persistent vectors. While the observer dependence of hermitian conjugation means that each observer sees her own 
basis vectors as real, she may find another observer's persistent basis vectors to be complex.

However, a different transformation applies to spacetime vectors such as acceleration. There the separate conjugations applied by the two observers ensure that the pure spatial vector seen by one observer remains real for the other and that a scalar part is generated. Thus, two distinct boost transformations for vectors ensue, one for persistent vectors such as the electric field giving a complex vector, and the other for instantaneous spacetime vectors such as the proper acceleration giving a real paravector. The covariant nature of the object must be recognized in order to know which transformation to apply.

To establish explicit transformations of the measurable coefficients, we can write the transformations between observers in terms of a proper basis of a single observer. We then find that the transformations do not depend on which observer's proper basis and conjugation are used. A single proper basis and conjugation (reversion) operation in APS can in fact be used to relate all inertial observers, even though they have different absolute frames in STA. This leads to the relative APS approach, in which all inertial observers share a single conjugation and a single proper basis. It is consistent with the principle of relativity that all inertial frames are equivalent and only relative motion and orientation of frames matters. The different covariant behavior of the proper acceleration and the electric field corresponds to the difference between spacetime vectors and spacetime bivectors, and in relative APS these are represented by real paravectors and biparavectors, respectively. APS can be interpreted both in terms of spatial vectors and their products, and in covariant terms, with Minkowski vectors of spacetime. The absolute version of APS emphasizes the first and the relative version the second. In both cases, one clearly identifies the elements of APS with measurables in $\mathrm{STA}^{+}$, and although in the presence of more than one observer the two versions invoke different mappings to $\mathrm{STA}^{+}$, we have shown them both to be two different approaches within a single coherent geometric algebra, namely APS.

The ability of APS to model relativistic processes as faithfully as STA, even though as a vector space it has only half the number of independent elements, is due mainly to the additional structure required by the absolute-frame representation of relativity in STA. APS, in contrast, does not model non-observable absolute frames but concentrates instead on measurable properties relative to an observer. A characteristic of the APS approach that also contributes to its efficiency is the double role of vector grades. Thus, a scalar might be a Lorentz invariant or the time component of a paravector, and a vector might be part 
of a paravector or part of a biparavector. In this regard, APS models relativistic quantities as humans commonly do. For example, the mass of a particle is both the Lorentz invariant length of its momentum and the time component (in units with $c=1$ ) of the momentum in its rest frame. This is naturally expressed in APS, where $m=m \mathbf{e}_{0}$ is the same object in both roles, whereas in STA the roles are played by distinct elements: $m \neq m \gamma_{0}$. This feature may be considered an extension of a basic attraction of working with geometric algebras instead of with separate vector spaces: in the algebra there is one zero element, and one does not need to distinguish the zero scalar from the zero vector or zero bivector.

\section{Acknowledgment}

One of us (WEB) thanks the Natural Sciences and Engineering Research Council of Canada for support of research.

[1] D. Hestenes, Spacetime Algebra, Gordon and Breach, New York 1966.

[2] D. Hestenes and G. Sobczyk, Clifford Algebra to Geometric Calculus: A Unified Language for Mathematics and Physics, D. Reidel, Dordrecht, 1984.

[3] D. Hestenes, New Foundations for Classical Mechanics, 2nd edn., Kluwer Academic, Dordrecht, 1999.

[4] D. Hestenes, Am. J. Phys. 71:104-121, 2003; 71:691-714, 2003.

[5] W. E. Baylis, editor, Clifford (Geometric) Algebra with Applications to Physics, Mathematics, and Engineering, Birkhäuser, Boston 1996.

[6] P. Lounesto, Clifford Algebras and Spinors, second edition, Cambridge University Press, Cambridge (UK) 2001.

[7] R. Abłamowicz and G. Sobczyk, eds., Lectures on Clifford Geometric Algebras, Birkhäuser, Boston, 2003.

[8] J. Snygg, Clifford Algebra, a Computational Tool for Physicists, Oxford U. Press, Oxford, 1997.

[9] K. Gürlebeck and W. Sprössig, Quaternions and Clifford Calculus for Physicists and Engineers, J. Wiley and Sons, New York, 1997. 
[10] C. Doran and A. Lasenby, Geometric Algebra for Physicists, Cambridge University Press, Cambridge (UK), 2003.

[11] D. Hestenes, J. Math. Phys. 15, 1768-1777 (1974).

[12] W. E. Baylis, Electrodynamics: A Modern Geometric Approach, Birkhäuser, Boston, 1999.

[13] W. E. Baylis and G. Jones, J. Phys. A (Math Gen) 22, 1-16; 17-29 (1989).

[14] W. E. Baylis, Am. J. Phys. 48, 918-925 (1980).

[15] G. Sobczyk, Phys. Letters A :45-48 Jul (1981).

[16] L. Silberstein, Phil. Mag. 23, 790-809 (1912).

[17] A. W. Conway, Phil. Mag. 24, 208 (1912).

[18] G. Sobczyk, Acta Phys. Pol., Vol.B12, 509-521 (1981). 